\title{
The use of simulation modelling in the analysis of seasonal constraints in a goat production system in southern Tanzania
}

\author{
C. R. C. Hendy ${ }^{1}$ and A. B. Carles ${ }^{2}$ \\ 'Natural Resources Institute, Chatham Maritime, Kent ME4 4TB \\ ${ }^{2}$ Department of Animal Production, University of Nairobi, PO Box 29053, Kabete, Kenya
}

\section{Introduction}

Marked reductions in breeding goat live weights and offspring growth to weaning were observed during the rainy season in a goat production system under traditional management in southern Tanzania. These reductions were associated with reduced survival to weaning and delays in subsequent rebreeding of females kidding in the rains, leading to significant reductions in overall productivity of these animals.

These effects were thought to be caused by seasonal restrictions in food availability (due to tethering and limited grazing times) and by increased helminth challenges in the rainy season, despite seasonal improvements in apparent forage abundance and diet quality.

An understanding of the relative influence of the factors contributing to seasonal effects was important in order to design appropriate interventions in helminth control and supplementary feeding.

\section{Methods}

The Texas A\&M Sheep and Goat Simulation Model is designed to simulate the live weights, reproductive performance, milk yields, offspring growth and helminth parasite infections of breeding female goats in response to inputs describing food supplies and helminth challenge. The model was calibrated to the prevailing environment, food supply, helminth challenge and genetic potential of the local goat in southern Tanzania, based on the results of extensive field recording of the production system. The model successfully reproduced the observed seasonal effects (Figure 1).

Simulated alternate removal of seasonal constraints, by allowing either unrestricted food supplies or by suppression of parasite infections, then enabled an assessment of the relative importance of these constraints.

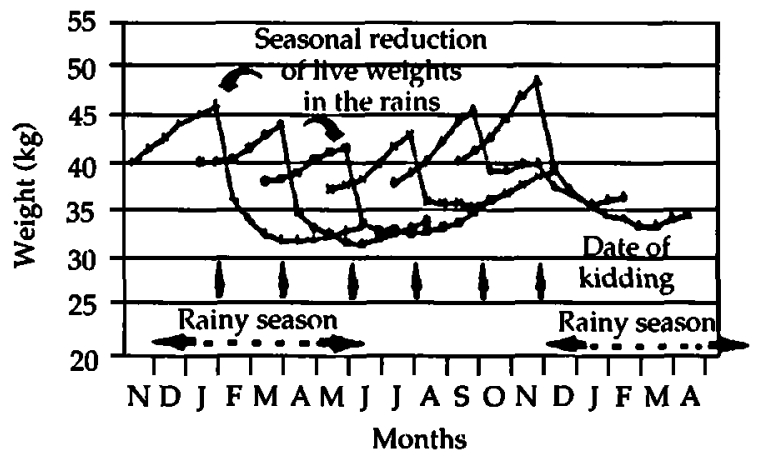

Figure 1 Simulations of observed pre- and post-partum weight changes of goats kidding in different seasons.

\section{Results}

Derestriction of food supplies alone did not notably reduce seasonal effects (Figure 2). The simulated removal of seasonal helminth challenges almost eliminated seasonal effects on breeding female live weights and offspring growth (Figure 3). Therefore, helminth challenge appeared to be the more important cause of seasonal effects.

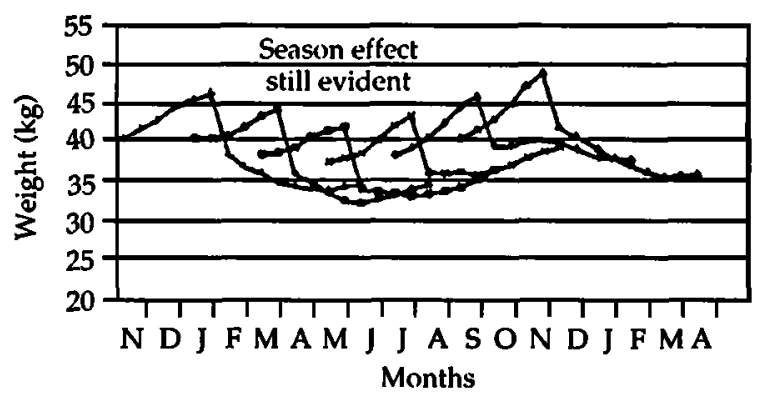

Figure 2 Breeding goat weight changes simulated with unrestricted food availability but no helminth control. Dates of kidding as for Figure 1. 


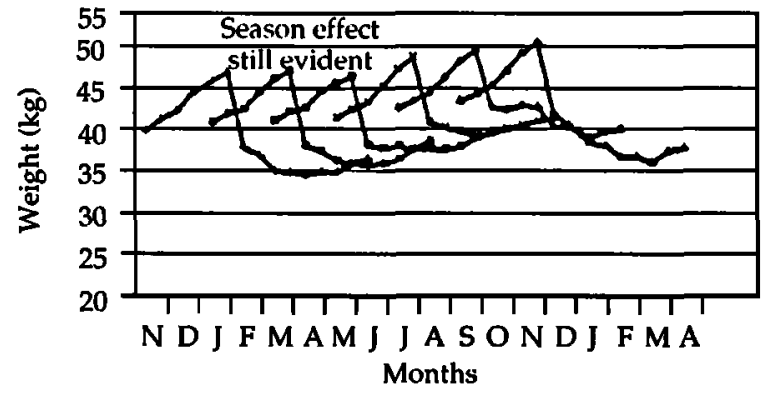

Figure 3 Breeding goat weight changes simulated with control of helminths but restricted food availability. Dates of kidding as for Figure 1.

Model simulations indicated that food intakes were limited by the restrictions of tethering in the early rainy season, but that this restriction only affected lactating animals with higher potential food demands.

Responses to unrestricted food availability in the rainy season were, however, limited unless helminth infections were also controlled.

Offspring growth was markedly improved by helminth control in the dam, but only marginally affected by derestriction of food supplies (Figure 4).

\section{Conclusions}

These findings illustrate the important effects of helminth infection on animal productivity in a subhumid environment.

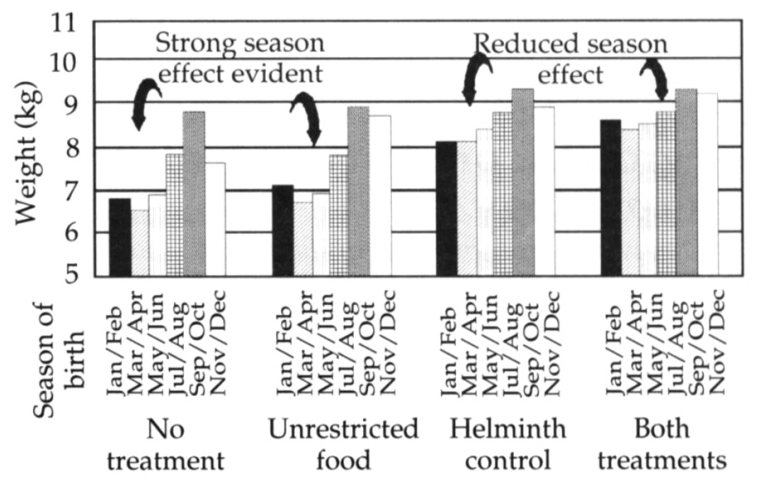

Figure 4 Simulated 90-day weights of kids born in different seasons to dams under different treatments.

They also suggest that improvements in feeding systems and responses to supplementary feeding are likely to be limited unless helminths are also controlled.

While these findings remain to be verified in the field, it is of interest to note how closely the model was able to simulate the observed season effects. If fully validated, simulation modelling can be a useful adjunct to field studies to improve understanding of systems and the design of interventions.

\section{Acknowledgements}

This work was funded by the Overseas Development Administration, UK. Dr H. D. Blackburn and Texas A\&M University kindly allowed use of the model. 